\title{
Diet quality and physical or comprehensive frailty among older adults
}

\author{
Daiki Watanabe ${ }^{1,2} \cdot$ Kayo Kurotani $^{1,3} \cdot$ Tsukasa Yoshida $^{1,2} \cdot$ Hinako Nanri $^{1} \cdot$ Yuya Watanabe $^{1,2,4} \cdot$ Heiwa Date $^{5}$. \\ Aya Itoi ${ }^{1,6}$. Chiho Goto $^{7} \cdot$ Kazuko Ishikawa-Takata $^{1,8} \cdot$ Misaka Kimura $^{2,9,10} \cdot$ Motohiko Miyachi $^{1,11}$. \\ Yosuke Yamada ${ }^{1,2}$ (1) Kyoto-Kameoka Study Group ${ }^{1}$
}

Received: 13 July 2021 / Accepted: 25 January 2022 / Published online: 13 February 2022

(c) The Author(s) 2022

\begin{abstract}
Purpose While the association between diet quality and mortality has been previously demonstrated, the association between frailty and diet quality has not been evaluated well. This study aimed to investigate the association between diet quality and prevalence of both physical and comprehensive frailty, using two validated tools, in a community-based cohort of older adults. Methods We conducted cross-sectional analyses using baseline data of 7022 participants aged $\geq 65$ years in the KyotoKameoka study. Diet quality was assessed by calculating the adherence scores to the Japanese Food Guide Spinning Top using a validated questionnaire; the participants were stratified into quartile groups based on these scores. Physical and comprehensive frailty was assessed using the Fried phenotype model-based Frailty Screening Index and the Kihon Checklist, respectively. Multivariable logistic regression and the restricted cubic spline model were used to calculate odds ratios (ORs) and their $95 \%$ confidence intervals (CIs) for associations between adherence scores and frailty prevalence.

Results Higher adherence scores signified a higher intake of vitamin C, vegetables, dairy products, and fruits. Physical and comprehensive frailty prevalence was 14.2 and $35.8 \%$, respectively. In a multivariable adjusted model, compared with the bottom adherence score quartile, the top quartile was associated with lower ORs of physical (OR 0.64; 95\% CI 0.52-0.80) and comprehensive frailty (OR $0.60 ; 95 \%$ CI $0.51-0.71$ ). These relationships were similar to results in the spline model. Conclusions This study shows an inverse dose-response relationship between diet quality and prevalence of both physical and comprehensive frailty in older adults.
\end{abstract}

Keywords Diet quality $\cdot$ Physical frailty $\cdot$ Comprehensive frailty $\cdot$ Japanese Food Guide Spinning Top · Cross-sectional study

\section{Introduction}

Frailty is a condition in which multiple physiological systems decline in function due to loss of homeostasis against the stress response $[1,2]$ and/or increased risk of adverse health outcomes [3] and is characterized by a multidimensional factor such as physical, psychosocial, and cognitive ability playing a part in its development [1-3]. It is an important global public health problem among older adults [3], and it has been reported that frailty is associated with adverse outcomes such as mortality [4, 5], fractures [5, 6], and falls [5]. Therefore, frailty must be prevented to ensure a healthy life expectancy without disease [7] and reduce the

Yosuke Yamada

yamaday@nibiohn.go.jp

Extended author information available on the last page of the article burden of healthcare-related costs [8]. Lifestyle care should shift towards more appropriate strategies that prevent frailty [1].

Diet quality is important for promoting health and lowering the risk of major diet-related diseases such as cancer [9-11]. Diet quality is measured using various adherence scores based on previous studies or national dietary guidelines $[10,11]$. The use of the Mediterranean diet intake [12-14], the Healthy Eating Index (HEI) [14, 15], or Dietary Approaches to Stop Hypertension (DASH) [14] may decrease the risk or odds ratios (ORs) of frailty. In Japan, the Japanese Food Guide Spinning Top was developed by the Ministry of Health, Labour, and Welfare and the Ministry of Agriculture, Forestry and Fisheries to promote healthy dietary habits [16]. While adherence to this guideline is inversely associated with mortality in middle-aged and older 
adults [17], it is unclear whether this diet quality is associated with frailty prevalence in older people.

Previous studies have reported an association between diet quality and defined frailty, using specific assessment tools [12-15]. Although the term "frailty" appears straightforward, its evaluation has varied among studies because many such assessment tools exist [18]. This can result in too much heterogeneity in predictive ability and classification. Therefore, measuring the association between diet quality and frailty prevalence needs to be done using a wellvalidated method. Notably, a previous study demonstrated that disease outcomes in patients admitted to the hospital with COVID-19 [19] were better predicted by frailty than by either age or comorbidity. This underlines the importance of assessing the impact of diet quality on frailty prevalence. Thus, we aimed to investigate the association between diet quality and prevalence of physical and comprehensive frailty using two validated assessment tools.

\section{Subjects and methods}

\section{Study population and baseline characteristic assessment}

The Kyoto-Kameoka study is an ongoing populationbased cohort study of community-dwelling residents aged $\geq 65$ years in Kameoka City, Japan. Residents were invited to participate in a baseline survey that included the Fried phenotype (FP) model-based Frailty Screening Index (FSI) and the Kihon Checklist (KCL) for the assessment of frailty status. The baseline survey took place on July 29, 2011, and details have been published elsewhere [20-27]. Our group conducted an additional survey, including the administration of the Food Frequency Questionnaire (FFQ), on February 14, 2012. Results of both surveys were collected via postal mail, and informed consent was obtained from participants along with their responses to these questionnaires. Information on medical history, socioeconomic status, health-related lifestyle behaviours, including smoking and drinking status, dietary habits, and physical activity, were obtained on each survey. The study was approved by the Ethics Committees of Kyoto Prefectural University of Medicine (RBMR-E-363), Kyoto University of Advanced Science (No. 20-1), and the National Institutes of Biomedical Innovation, Health and Nutrition (NIBIOHN-76-2). This study has been carried out in accordance with The Code of Ethics of the World Medical Association (Declaration of Helsinki) and Strengthening the Reporting of Observational Studies in Epidemiology (STROBE)-Nutritional Epidemiology criteria [28].

Out of the total number of participants at baseline $(n=13,294), 8319$ responded to our additional survey. Those with an incomplete KCL or FSI $(n=1089)$, who had selfreported "needed long-term care" $(n=122)$, and who had extremely high or low energy intakes (standard deviation [SD] of 3 from the mean energy intake) [23] in each sex group ( $n=86$ ), were excluded. Ultimately, 7022 participants were included in the present analysis.

\section{Dietary assessment}

Dietary nutrient and food intakes were estimated using the FFQ [24, 25, 29-31]. We have previously validated the FFQ using dietary records $[24,29,31]$ and doubly labelled water (DLW) methods [25, 32] in a subpopulation of participants of the Kyoto-Kameoka study. On the FFQ, participants were asked to report their intake frequency of 47 food and beverage items over the past year. Food and nutrient intakes were then calculated using the frequency of appearance of each food and beverage category and the portion size for each sex group [30] or using a program based on the Standard Tables of Food Composition in Japan [33]. Energy intake was calibrated using our previously developed equation that uses total energy expenditure (as measured by the DLW method) [25]. The equation used for this calculation attenuates the impact of age, sex, and body mass index (BMI)-related selfreporting bias on estimated energy intake in older adults, assessed via the FFQ [32].

\section{Calculation of diet quality score}

To assess diet quality, we calculated participants' adherence scores from the Japanese Food Guide Spinning Top (the food-based Japanese dietary guidelines) [16]. The details of this measure have been published elsewhere [16, 17, 27, 34]. Recommended serving amounts for each category and recommended total energy intake are specified according to sex, age, and level of physical activity (i.e., participation in manual labour or walking for at least $1 \mathrm{~h}$ a day were labelled as moderately physically active; other activity levels were labelled as sedentary) [16, 17, 27, 34]. We calculated the number of servings according to the food guide criteria. One serving of each was defined as follows: a grain dish (40 $\mathrm{g}$ of carbohydrates), vegetable dish (weight, $70 \mathrm{~g}$ ), fish or meat dish (6 $\mathrm{g}$ of protein), milk (100 mg calcium), and fruit (weight of $100 \mathrm{~g}$ ) [16, 17, 27, 34]. We determined food guide adherence scores using a method described by Kurotani et al. [17, 27]. To calculate the scores for each component, food items were categorised as follows: grain dishes (rice, bread, and noodles); vegetable dishes (potatoes, pump$\mathrm{kin} / \mathrm{squash}$, carrots, broccoli, green-leaved vegetables, other green/yellow vegetables, cabbage, daikon (Japanese radish), kiriboshi-daikon (dry strips of Japanese radish), burdock, bamboo shoots, other vegetables, mushrooms, seaweed, peanuts, and almonds); fish and meat dishes (tofu (soybean 
curd), natto (fermented soybeans), soybeans, eggs, chicken, beef, pork, liver, ham, sausage, bacon, salami sausage, fish, bone-edible small fish, canned tuna, cuttlefish, squid, octopus, shrimp, crab, shellfish, fish eggs, fish paste products, ganmodoki (fried tofu paste), and nama-age (fried tofu); [soybeans were included in the fish and meat dish category, based on their high-protein nutrient profile]); milk products (milk and yogurt); fruits (citrus and other fruits); and snacks and alcoholic beverages (western-style confectioneries, Japanese-style confectioneries, and alcohol). In addition to the Japanese Food Guide Spinning Top criteria, we calculated adherence scores based on the intake ratio of white to red meat as a component of the score [17, 27]. White meat included chicken, fish, bone-edible small fish, canned tuna, cuttlefish, squid, octopus, shrimp, crab, shellfish, fish eggs, and fish paste products. Red meat included beef, pork, liver, ham, sausage, bacon, and salami sausage. Each component was given a score between 0 and 10 , and to obtain a total score that ranged from 0 (lowest adherence) to 80 (highest adherence), all the component scores were summed.

\section{Definition of frailty}

Frailty was assessed using the FP model-based five-item FSI [7, 21-23] and the 25-item KCL [21-23, 35, 36], both of which have been validated according to the risk of LongTerm Care Insurance Certification [7, 35]. Physical frailty was evaluated using the FSI, focusing on the physical aspects, and was defined as a score of 3 or more points out of a maximum of 5 (weight loss, slow gait speed, cognition, exhaustion, and low physical activity) [7, 21-23]. Comprehensive frailty was evaluated using the KCL. It includes cognitive and social aspects, as well as physical factors, and was defined as a score of 7 or higher out of a possible 25 points [21-23, 35, 36]. Additionally, we investigated possible associations between the FSI and KCL subdomains and the adherence score. The KCL consists of seven subdomains: instrumental activity of daily living (IADL) disability, physical, nutrition, oral, social, cognitive, and depression. Detailed methods of the subdomain investigation are provided elsewhere [26].

\section{Statistical analysis}

Adherence scores to the Japanese Food Guide Spinning Top were divided into quartiles (Qs). Continuous and categorical variables are shown as means with SDs and numbers with percentages, respectively. Where information pertaining to the BMI $(n=35)$, alcohol status $(n=102)$, smoking status $(n=385)$, physical activity $(n=359)$, family structure $(n=468)$, socioeconomic status $(n=235)$, education attainment $(n=645)$, denture use $(n=82)$, or medications $(n=387)$ was missing, we performed multiple imputation on five data sets, using chained equations from R statistical software [37] to prevent a systematic error appearing due to selection bias. Missing data were assumed to be missing at random. Nutrient intake was adjusted for energy intake per $1000 \mathrm{kcal}$ via the nutrient density method, using uncalibrated energy intake [38]. These values are shown as the median of each quartile group. The association between adherence score and nutrient intake was confirmed using Spearman's correlation analysis.

The number of frailty cases is shown as a case (\%). Multivariate logistic regression analysis was performed, considering several baseline covariate variables. Multivariate adjusted analyses were verified by two models: Model 1 was adjusted for age (continuous), sex (female or male), and population density ( $\geq 1000$ or $<1000$ people $\left./ \mathrm{km}^{2}\right)$. In model 2 , in addition to the factors adjusted for in model 1 , we adjusted for body mass index (continuous), physical activity (yes or no), denture use (yes or no), smoking status (never smoker, past smoker, or current smoker), alcohol intake status (every day, sometimes, seldom, or never), educational attainment $(<9,10-12$, or $\geq 13$ years), medication use (continuous), living alone (yes or no), socioeconomic status (high or low), green tea consumption (frequency), coffee consumption (frequency), and history of the disease (hypertension, diabetes, dyslipidaemia, heart disease, and stroke; yes or no). These variables were selected according to covariates used in a previous study [21-23, 26]. ORs for frailty are presented as OR (95\% confidence interval [CI]), using the first quartile group (lowest diet quality) as the referent group. Diet quality is better among women [17] and affluent people [39]. Thus there is a possibility of evaluating primarily sex or socioeconomic status-specific influences when ranking individuals according to adherence scores. Therefore, subgroup analyses were performed separately by sex and socioeconomic status (high or low). Additionally, we evaluated possible associations between the adherence score and the subdomains that were evaluated using the FSI and KCL.

To evaluate the curve association of diet quality and frailty prevalence, we used a restricted cubic spline model considering three data points (5th, 50th, and 95th percentiles), based on the distribution of the recommended adherence score. Because the data were sparse, we truncated the analysis at 29 points ( $1 \%$ of the distribution) [22]. We calculated the ORs for frailty prevalence associated with adherence score, using the 43 points of first quartile value as the reference in the restricted cubic spline model [21]. Moreover, we considered the association between frailty prevalence and the adherence score of each component of the Japanese Food Guide Spinning Top. A two-sided $p$ value $<0.05$ was considered significant. Linear trends were computed by treating adherence score exposure as a continuous variable. Statistical analyses were performed using STATA MP, 
version 15.0 (Stata Corp LP, College Station, TX), and/or R software 3.4.3 (R Core Team, Vienna, Austria).

\section{Results}

The mean score on the adherence to the Japanese Food Guide Spinning Top was 54.3 (SD 8.4). Compared to participants with lower adherence scores, participants with higher scores were likelier to be women, live alone, have higher educational attainment and socioeconomic status, and have a history of hyperlipidaemia. They were less likely to be current smokers and alcohol drinkers (Table 1).

The associations between adherence scores and nutrient and food intake are shown in Table 2. Adherence scores were moderately correlated to the intake of the following: vitamin C $(r=0.46)$, vegetables $(r=0.42)$, fruits $(r=0.56)$, and dairy products $(r=0.46)$. Participants with higher adherence scores tended to have a higher intake of fat, folate, and calcium $(\geq r=0.30)$. Therefore, participants with higher adherence scores have characteristics of high vitamin $C$, vegetables, fruits, and dairy products intake. However, energy, protein, and carbohydrate intake were not observed to be significantly different.

Table 3 shows the associations between adherence score and physical and comprehensive frailty prevalence. Physical and comprehensive frailty prevalence was 14.2 and $35.8 \%$, respectively. We found that adherence score was negatively associated with the OR of prevalence of physical frailty, as defined by the FP model, after adjusting for baseline potential confounding factors $(\mathrm{Q} 1$ : reference; $\mathrm{Q} 2$ : OR $0.87 ; 95 \%$ CI 0.72-1.06; Q3: OR 0.84; 95\% CI 0.69-1.03; Q4: OR $0.64 ; 95 \%$ CI $0.52-0.80$ ); $P$ for trend $<0.001)$. Similar findings were observed with comprehensive frailty. In subgroup analyses, comparable results were observed in both the sex and socioeconomic status-stratified models (Supplemental Tables 1 and 2). Furthermore, we demonstrated that the adherence score was negatively associated with ORs for the prevalence of slow gait speed, cognition, exhaustion, and low physical activity, after evaluation using the FP model subdomains (Supplementary Table S3). These indicated a negative relationship with ORs for the prevalence of IADL disability, social frailty, cognitive frailty, and depression after evaluation using the KCL subdomains (Supplementary Table S4).

Table 1 Baseline participant characteristics according to quartile of adherence to the Japanese Food Guide Spinning Top

\begin{tabular}{|c|c|c|c|c|c|c|c|c|c|c|c|}
\hline \multirow[b]{3}{*}{ Age [years] $]^{a}$} & \multirow{2}{*}{\multicolumn{2}{|c|}{ Total $(n=7022)$}} & \multicolumn{8}{|c|}{ Quartile of the Japanese Food Guide Spinning Top score } & \multirow{3}{*}{$\begin{array}{c}p \text { value } \\
\mathbf{0 . 0 3 9}\end{array}$} \\
\hline & & & \multicolumn{2}{|c|}{$\mathrm{Q} 1(n=1756)$} & \multicolumn{2}{|c|}{$\mathrm{Q} 2(n=1756)$} & \multicolumn{2}{|c|}{$\mathrm{Q} 3(n=1755)$} & \multicolumn{2}{|c|}{$\mathrm{Q} 4(n=1755)$} & \\
\hline & 73.3 & $(6.1)$ & 73.5 & $(6.2)$ & 73.2 & $(6.2)$ & 73.0 & $(6.0)$ & 73.4 & $(6.0)$ & \\
\hline Women $[n(\%)]^{\mathrm{b}}$ & 3709 & $(52.8)$ & 551 & $(31.4)$ & 874 & $(49.8)$ & 1044 & $(59.5)$ & 1240 & $(70.7)$ & $<0.001$ \\
\hline $\mathrm{PD} \geq 1000$ people $/ \mathrm{km}^{2}[n(\%)]^{\mathrm{b}}$ & 3232 & $(46.0)$ & 738 & $(42.0)$ & 781 & $(44.5)$ & 814 & $(46.4)$ & 899 & $(51.2)$ & $<0.001$ \\
\hline BMI $\left[\mathrm{kg} / \mathrm{m}^{2}\right]^{\mathrm{a}}$ & 22.6 & (3.0) & 22.7 & (3.1) & 22.7 & (3.1) & 22.7 & $(3.0)$ & 22.3 & (2.9) & $<0.001$ \\
\hline Alcohol drinker $[n(\%)]^{\mathrm{b}}$ & 4595 & $(65.4)$ & 1309 & $(74.5)$ & 1174 & $(66.9)$ & 1077 & $(61.4)$ & 1035 & $(59.0)$ & $<0.001$ \\
\hline Current smoker $[n(\%)]^{\mathrm{b}}$ & 754 & $(10.7)$ & 335 & $(19.1)$ & 212 & $(12.1)$ & 115 & (6.6) & 92 & (5.3) & $<0.001$ \\
\hline $\operatorname{MVPA}[n(\%)]^{\mathrm{b}}$ & 3114 & $(44.4)$ & 746 & $(42.5)$ & 802 & $(45.7)$ & 788 & $(44.9)$ & 778 & $(44.3)$ & 0.268 \\
\hline Living alone $[n(\%)]^{\mathrm{b}}$ & 789 & $(11.2)$ & 171 & $(9.7)$ & 167 & $(9.5)$ & 214 & $(12.2)$ & 237 & $(13.5)$ & $<0.001$ \\
\hline $\operatorname{HSES}[n(\%)]^{\mathrm{b}}$ & 2394 & $(34.1)$ & 507 & (28.9) & 546 & $(31.1)$ & 623 & $(35.5)$ & 718 & $(40.9)$ & $<0.001$ \\
\hline Education $\geq 13$ y $[n(\%)]^{\mathrm{b}}$ & 1576 & $(22.4)$ & 359 & $(20.4)$ & 386 & $(22.0)$ & 414 & $(23.6)$ & 417 & $(23.8)$ & $<0.001$ \\
\hline Denture use $[n(\%)]^{\mathrm{b}}$ & 4212 & $(60.0)$ & 1099 & $(62.6)$ & 1074 & $(61.2)$ & 1015 & $(57.8)$ & 1024 & $(58.4)$ & 0.010 \\
\hline No medication $[n(\%)]^{\mathrm{b}}$ & 1575 & $(22.4)$ & 405 & $(23.1)$ & 431 & $(24.5)$ & 367 & $(20.9)$ & 372 & $(21.2)$ & $<0.001$ \\
\hline Hypertension $[n(\%)]^{\mathrm{b}}$ & 2731 & $(38.9)$ & 656 & $(37.4)$ & 677 & $(38.6)$ & 709 & $(40.4)$ & 689 & $(39.3)$ & 0.308 \\
\hline Stroke $[n(\%)]^{\mathrm{b}}$ & 251 & (3.6) & 71 & $(4.0)$ & 78 & $(4.4)$ & 54 & (3.1) & 48 & (2.7) & 0.020 \\
\hline Heart disease $[n(\%)]^{\mathrm{b}}$ & 869 & $(12.4)$ & 247 & $(14.1)$ & 228 & $(13.0)$ & 175 & $(10.0)$ & 219 & $(12.5)$ & 0.002 \\
\hline Diabetes $[n(\%)]^{\mathrm{b}}$ & 736 & $(10.5)$ & 193 & $(11.0)$ & 190 & $(10.8)$ & 180 & (10.3) & 173 & (9.9) & 0.677 \\
\hline Hyperlipidemia $[n(\%)]^{\mathrm{b}}$ & 711 & $(10.1)$ & 112 & $(6.4)$ & 150 & $(8.5)$ & 193 & $(11.0)$ & 256 & (14.6) & $<0.001$ \\
\hline
\end{tabular}

Bold $p$ values are statistically significant $(p<0.05)$. Data of participants with missing values underwent multiple imputation: body mass index $(n=35)$; alcohol status $(n=102)$; smoking status $(n=385)$; physical activity $(n=359)$; family structure $(n=468)$; socioeconomic status $(n=235)$; education attainment $(n=645)$; denture use $(n=82)$; medications $(n=387)$. BMI, body mass index; HSES, high socioeconomic status; MVPA, moderate to vigorous physical activity; PD, population density. Q1 through Q4 includes Japanese Food Guide Spinning Top scores of $<49.5,49.5-54.8,54.9-60.1$, and $\geq 60.2$

${ }^{a}$ Continuous values are shown as mean (standard deviation) and are analyzed using variance analysis

${ }^{\mathrm{b}}$ Categorical values are shown as number (percentage) and are analyzed using the Chi-square test. MVPA means reported MVPA exercise habits from a questionnaire 
Table 2 Association between energy and nutrient intake and adherence to the Japanese Food Guide Spinning Top

\begin{tabular}{|c|c|c|c|c|c|}
\hline & \multicolumn{4}{|c|}{ Quartile of the Japanese Food Guide spinning top score } & \multirow[t]{2}{*}{$r^{\mathrm{a}}$} \\
\hline & $\mathrm{Q} 1(n=1756)$ & $\mathrm{Q} 2(n=1756)$ & $\mathrm{Q} 3(n=1755)$ & $\mathrm{Q} 4(n=1755)$ & \\
\hline \multicolumn{6}{|l|}{ Nutrients } \\
\hline Calibrated EI $[\mathrm{kcal} / \mathrm{day}]^{\mathrm{b}}$ & 2294 & 2151 & 2047 & 1995 & -0.23 \\
\hline Uncalibrated EI [kcal/day] & 1832 & 1722 & 1702 & 1697 & -0.05 \\
\hline Protein [\% energy/day] & 10.7 & 12.0 & 12.4 & 12.7 & 0.28 \\
\hline Fat [\% energy/day] & 19.1 & 23.3 & 25.9 & 28.7 & 0.35 \\
\hline Carbohydrate [\% energy/day] & 55.9 & 56.0 & 55.7 & 55.6 & -0.01 \\
\hline SFA [g/1000 kcal/day] & 5.4 & 6.4 & 6.9 & 7.4 & 0.26 \\
\hline MUFA [g/1000 kcal/day] & 8.2 & 9.6 & 10.7 & 12.0 & 0.29 \\
\hline PUFA [g/1000 kcal/day] & 7.0 & 8.3 & 8.9 & 9.8 & 0.27 \\
\hline n-6 PUFA [g/1000 kcal/day] & 6.0 & 7.3 & 7.8 & 8.7 & 0.28 \\
\hline n-3 PUFA [g/1000 kcal/day] & 1.0 & 1.2 & 1.3 & 1.5 & 0.29 \\
\hline Cholesterol [mg/1000 kcal/day] & 108 & 119 & 122 & 125 & 0.12 \\
\hline Vitamin A [ $\mu \mathrm{g}$ RE $/ 1000 \mathrm{kcal} / \mathrm{day}]^{\mathrm{c}}$ & 393 & 477 & 520 & 492 & 0.11 \\
\hline Vitamin D [ $\mu \mathrm{g} / 1000 \mathrm{kcal} / \mathrm{day}]$ & 2.3 & 2.5 & 2.5 & 2.4 & 0.03 \\
\hline$\alpha$-Tocopherol [mg/1000 kcal/day] & 4.5 & 5.4 & 5.8 & 6.4 & 0.28 \\
\hline Folate $[\mu \mathrm{g} / 1000 \mathrm{kcal} / \mathrm{day}]$ & 143 & 174 & 191 & 216 & 0.34 \\
\hline Vitamin C [mg/1000 kcal/day] & 41 & 52 & 61 & 75 & 0.46 \\
\hline Sodium [mg/1000 kcal/day] & 897 & 972 & 978 & 981 & 0.08 \\
\hline Potassium [mg/1000 kcal/day] & 978 & 1101 & 1161 & 1226 & 0.29 \\
\hline Iron $[\mathrm{mg} / 1000 \mathrm{kcal} / \mathrm{day}]$ & 3.2 & 3.7 & 3.8 & 4.0 & 0.19 \\
\hline Calcium [mg/1000 kcal/day] & 224 & 271 & 309 & 331 & 0.31 \\
\hline Total dietary fiber $[\mathrm{g} / 1000 \mathrm{kcal} / \mathrm{day}]$ & 5.0 & 5.8 & 6.1 & 6.6 & 0.28 \\
\hline Alcohol [g/1000 kcal/day] & 0.8 & 0.0 & 0.0 & 0.0 & -0.22 \\
\hline \multicolumn{6}{|l|}{ Food } \\
\hline Grains [g/1000 kcal/day] & 272 & 262 & 253 & 247 & -0.15 \\
\hline Vegetables [g/1000 kcal/day] & 59 & 85 & 99 & 126 & 0.42 \\
\hline Fruits [g/1000 kcal/day] & 13 & 25 & 44 & 70 & 0.56 \\
\hline Dairies [g/1000 kcal/day] & 6 & 42 & 81 & 95 & 0.46 \\
\hline Red meats [g/1000 kcal/day] & 11 & 12 & 12 & 9 & -0.03 \\
\hline White meats [g/1000 kcal/day] & 34 & 44 & 46 & 47 & 0.19 \\
\hline Confectionery [g/1000 kcal/day] & 10 & 9 & 9 & 8 & -0.13 \\
\hline
\end{tabular}

All values are shown medians or correlation coefficients. Nutrient intake was adjusted for energy intake via the nutrient density method, using uncalibrated energy intake. Values are shown as medians in each quartile group. Q1 through Q4 included Japanese Food Guide Spinning Top scores of $<49.5,49.5-54.8,54.9-60.1$, and $\geq 60.2$. EI, energy intake; MUFA, monounsaturated fatty acid; PUFA, polyunsaturated fatty acid; SFA, saturated fatty acid

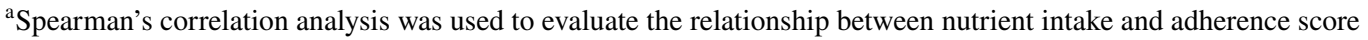

${ }^{\mathrm{b}}$ Calibrated EI was calculated using an equation developed by the authors

${ }^{\mathrm{c}} \mathrm{Sum}$ of retinol, $\beta$-carotene/12, $\alpha$-carotene/24, and cryptoxanthin/24

To evaluate the curve of the association between frailty prevalence and adherence score, we used a restricted cubic spline model (Fig. 1). When the first quartile (43 points) for adherence score was used as the reference, the shape of the curve showed a modest and negative association between adherence score and prevalence of each type of frailty, defined by the FP model or the KCL, up to approximately 55 points. This is the average adherence score of the current study population. Beyond this, an adherence score of up to approximately 76 points showed a strong dose-dependent negative association with frailty prevalence.

The association of the adherence score of each component with physical frailty prevalence is shown in Table 4 . After adjusting for confounders, the top adherence score quartile revealed a significant association with a lower OR of physical frailty (reference, lowest quartile of adherence scores): vegetable dishes (OR 0.86 [95\% CI 0.70-1.06], adjusted $p$ for trend $=0.041)$ and milk (OR $0.79[95 \% \mathrm{CI}$ 
Table 3 Multivariable adjusted odds ratios and 95\% confidence intervals of the prevalence of physical and comprehensive frailty according to Japanese Food Guide Spinning Top adherence score

\begin{tabular}{|c|c|c|c|c|c|c|c|c|c|c|c|}
\hline \multirow[b]{3}{*}{ Mean (SD) score } & \multicolumn{8}{|c|}{ Quartile of the Japanese Food Guide Spinning Top score } & \multirow{2}{*}{\multicolumn{2}{|c|}{10 points increment }} & \multirow[t]{3}{*}{$p$ for trend } \\
\hline & \multicolumn{2}{|c|}{ Q1 $(n=1756)$} & \multicolumn{2}{|c|}{$\mathrm{Q} 2(n=1756)$} & \multicolumn{2}{|c|}{$\mathrm{Q} 3(n=1755)$} & \multicolumn{2}{|c|}{$\mathrm{Q} 4(n=1755)$} & & & \\
\hline & 43.2 & $(5.9)$ & 52.3 & $(1.6)$ & 57.5 & $(1.5)$ & 64.1 & $(3.0)$ & & & \\
\hline \multicolumn{12}{|l|}{ Physical frailty } \\
\hline Case $[n(\%)]$ & 302 & $(17.2)$ & 259 & $(14.7)$ & 241 & $(13.7)$ & 196 & $(11.2)$ & & & \\
\hline Model $1^{\mathrm{b}}$ & 1.00 & (Ref) & 0.81 & $(0.68-0.98)$ & 0.75 & $(0.62-0.90)$ & 0.56 & $(0.45-0.68)$ & 0.79 & $(0.71-0.87)$ & $<0.001$ \\
\hline Model $2^{\mathrm{c}}$ & 1.00 & $(\operatorname{Ref})$ & 0.87 & $(0.72-1.06)$ & 0.84 & $(0.69-1.03)$ & 0.64 & $(0.52-0.80)$ & 0.85 & $(0.76-0.93)$ & $<0.001$ \\
\hline \multicolumn{12}{|c|}{ Comprehensive frailty } \\
\hline Case $[n(\%)]$ & 744 & $(42.4)$ & 658 & $(37.5)$ & 593 & $(33.8)$ & 519 & $(29.6)$ & & & \\
\hline Model $1^{\mathrm{b}}$ & 1.00 & (Ref) & 0.79 & $(0.68-0.91)$ & 0.66 & $(0.57-0.76)$ & 0.49 & $(0.42-0.57)$ & 0.70 & $(0.64-0.76)$ & $<0.001$ \\
\hline Model $2^{\mathrm{c}}$ & 1.00 & (Ref) & 0.86 & $(0.73-1.00)$ & 0.77 & $(0.66-0.90)$ & 0.60 & $(0.51-0.71)$ & 0.79 & $(0.72-0.86)$ & $<0.001$ \\
\hline
\end{tabular}

All values are shown means (SDs), numbers (\%), or relative ORs (95\% CI). All estimates were derived from a multivariable logistic regression model. Physical and comprehensive frailty was assessed using the validated Fried phenotype model-based Frailty Screening Index and the Kihon Checklist. Bold $p$ values are statistically significant $(p<0.05)$. Q1 through Q4 included Japanese Food Guide Spinning Top scores of $<49.5$, $49.5-54.8,54.9-60.1$, and $\geq 60.2$

CI, confidence interval; OR, odds ratio; Ref, reference; SD, standard deviation

${ }^{a}$ Linear trend $p$ values were calculated with the likelihood ratio test using continuous variables of adherence scores

${ }^{b}$ Model 1 was adjusted for age (continuous), sex (female or male), and population density ( $\geq 1000$ or $<1000$ people $/ \mathrm{km}^{2}$ )

${ }^{\mathrm{c}}$ Model 2 was Model 1 with mutual adjustments for body mass index (continuous), physical activity (yes or no), denture use (yes or no), smoking status (never smoker, past smoker, and current smoker), alcohol intake status (every day, sometimes, seldom, or never), educational attainment $(<9,10-12$, or $\geq 13$ years), medication use (continuous), living alone (yes or no), socioeconomic status (high or low), green tea consumption (frequency), coffee consumption (frequency), and history of disease (hypertension, diabetes, dyslipidaemia, heart disease, and stroke; yes or no)

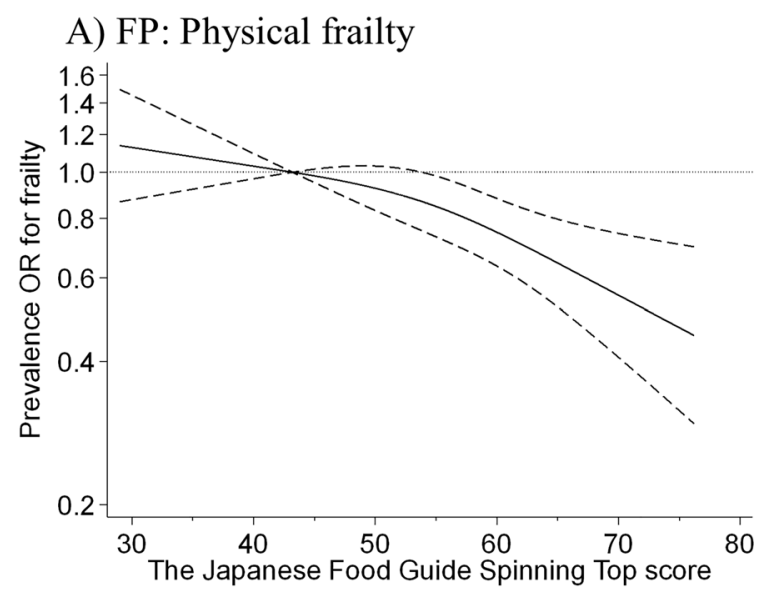

Fig. 1 Relationship between the Japanese Food Guide Spinning Top adherence score and frailty, based on A fried phenotype (FP) model and $\mathbf{B}$ the Kihon Checklist (KCL), using a restricted cubic spline logistic regression model. Frailty, according to the FP model-based self-administered frailty screening index (FSI), was defined as a score of $\geq 3$ out of 5 points. Frailty, according to the KCL, was defined as a score $\geq 7$ out of 25 points. Solid lines represent odds ratios (ORs), and dashed lines represent $95 \%$ confidence intervals (CIs). We calculated ORs for frailty prevalence using a first quartile value of 43

$0.65-0.97]$, adjusted $p$ for trend $=0.003$ ). Similar findings were observed with comprehensive frailty as defined by the KCL (Supplemental Tables 5). Furthermore, a high score on

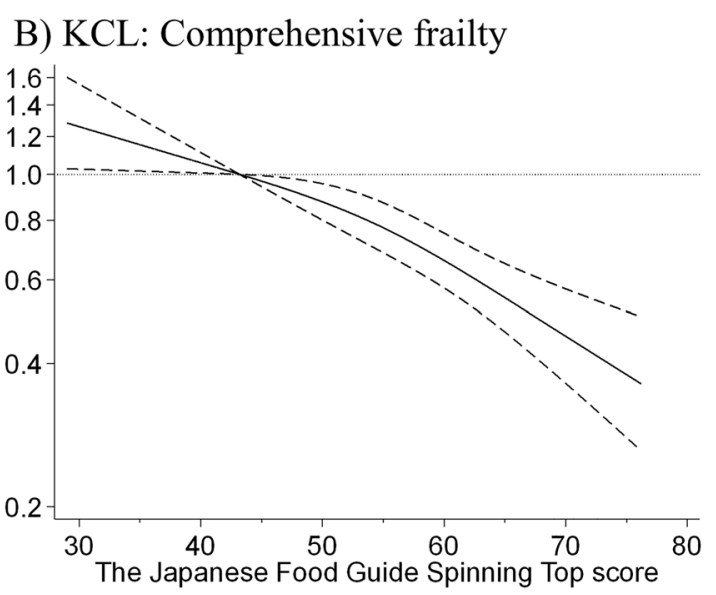

points as the reference. This analysis included 6954 participants. We estimated that $p<0.05$ when $95 \%$ CI of the OR did not exceed 1.00 , and $p \geq 0.05$ when $95 \% \mathrm{CI}$ of the OR exceeded 1.00 . Analyses were adjusted for age, sex, population density, body mass index, physical activity, denture use, smoking status, alcohol intake status, educational attainment, medication use, living alone, socioeconomic status, green tea consumption, coffee consumption, and history of disease (hypertension, diabetes, dyslipidaemia, heart disease, and stroke)

adherence to fruits and snacks and alcoholic beverages was associated with a low prevalence of comprehensive frailty, while a high score on adherence to energy intake showed a 
Table 4 Multivariable adjusted odds ratios and 95\% confidence intervals of the prevalence of physical frailty according to adherence score of each component on the Japanese Food Guide Spinning Top

\begin{tabular}{|c|c|c|c|c|c|c|c|c|c|c|c|}
\hline \multirow{3}{*}{ Grain dishes } & \multicolumn{8}{|c|}{ Quartile of the Japanese Food Guide Spinning Top score } & \multicolumn{2}{|c|}{1 points increment } & \multirow[t]{2}{*}{$p$ for trend } \\
\hline & \multicolumn{2}{|c|}{$\mathrm{Q} 1(n=1756)$} & \multicolumn{2}{|c|}{$\mathrm{Q} 2(n=1756)$} & \multicolumn{2}{|c|}{$\mathrm{Q} 3(n=1755)$} & \multicolumn{2}{|c|}{$\mathrm{Q} 4(n=1755)$} & & & \\
\hline & & & & & & & & & & & \\
\hline Case $[n(\%)]$ & 250 & $(14.2)$ & 257 & $(14.6)$ & 243 & $(13.8)$ & 248 & $(14.1)$ & & & \\
\hline Model $1^{\mathrm{b}}$ & 1.00 & (Ref) & 1.01 & (0.84 to 1.23$)$ & 0.92 & $(0.76-1.11)$ & 0.88 & $(0.73-1.07)$ & 0.97 & $(0.95-1.00)$ & 0.079 \\
\hline Model $2^{\mathrm{c}}$ & 1.00 & (Ref) & 1.02 & $(0.84-1.24)$ & 0.92 & $(0.76-1.12)$ & 0.86 & $(0.70-1.04)$ & 0.98 & $(0.95-1.00)$ & 0.098 \\
\hline \multicolumn{12}{|c|}{ Vegetable dishes } \\
\hline Case $[n(\%)]$ & 273 & $(15.5)$ & 265 & $(15.1)$ & 231 & $(13.2)$ & 229 & $(13.0)$ & & & \\
\hline Model $1^{\mathrm{b}}$ & 1.00 & (Ref) & 0.97 & $(0.81-1.17)$ & 0.79 & $(0.65-0.96)$ & 0.73 & $(0.60-0.89)$ & 0.95 & $(0.92-0.97)$ & $<0.001$ \\
\hline Model $2^{\mathrm{c}}$ & 1.00 & (Ref) & 1.03 & $(0.85-1.25)$ & 0.87 & $(0.71-1.07)$ & 0.86 & $(0.70-1.06)$ & 0.97 & $(0.95-0.99)$ & 0.041 \\
\hline \multicolumn{12}{|c|}{ Fish and meat dishes } \\
\hline Case $[n(\%)]$ & 269 & (15.3) & 266 & $(15.1)$ & 225 & $(12.8)$ & 238 & (13.6) & & & \\
\hline Model $1^{\mathrm{b}}$ & 1.00 & (Ref) & 1.07 & $(0.89-1.29)$ & 0.92 & $(0.76-1.12)$ & 0.99 & $(0.82-1.20)$ & 1.00 & $(0.98-1.02)$ & 0.801 \\
\hline Model $2^{\mathrm{c}}$ & 1.00 & (Ref) & 1.02 & $(0.85-1.24)$ & 0.87 & $(0.71-1.06)$ & 0.95 & $(0.78-1.15)$ & 0.99 & $(0.97-1.01)$ & 0.294 \\
\hline \multicolumn{12}{|l|}{ Milk } \\
\hline Case $[n(\%)]$ & 307 & $(17.5)$ & 264 & $(15.0)$ & 204 & (11.6) & 223 & (12.7) & & & \\
\hline Model $1^{\mathrm{b}}$ & 1.00 & (Ref) & 0.90 & $(0.75-1.09)$ & 0.66 & $(0.54-0.80)$ & 0.71 & $(0.59-0.86)$ & 0.96 & $(0.94-0.98)$ & $<0.001$ \\
\hline Model $2^{c}$ & 1.00 & (Ref) & 0.92 & $(0.77-1.11)$ & 0.71 & $(0.58-0.86)$ & 0.79 & $(0.65-0.97)$ & 0.97 & $(0.95-0.99)$ & 0.003 \\
\hline \multicolumn{12}{|l|}{ Fruits } \\
\hline Case $[n(\%)]$ & 300 & $(17.1)$ & 246 & $(14.0)$ & 221 & $(12.6)$ & 231 & $(13.2)$ & & & \\
\hline Model $1^{\mathrm{b}}$ & 1.00 & (Ref) & 0.79 & $(0.65-0.95)$ & 0.67 & $(0.55-0.81)$ & 0.68 & $(0.56-0.83)$ & 0.96 & $(0.93-0.98)$ & $<0.001$ \\
\hline Model $2^{c}$ & 1.00 & (Ref) & 0.87 & $(0.72-1.06)$ & 0.78 & $(0.64-0.95)$ & 0.83 & $(0.68-1.02)$ & 0.98 & $(0.96-1.00)$ & 0.109 \\
\hline \multicolumn{12}{|l|}{ Total energy } \\
\hline Case $[n(\%)]$ & 230 & (13.1) & 243 & (13.8) & 278 & $(15.8)$ & 247 & $(14.1)$ & & & \\
\hline Model $1^{\mathrm{b}}$ & 1.00 & (Ref) & 1.31 & $(1.05-1.62)$ & 1.44 & $(1.14-1.82)$ & 1.17 & $(0.91-1.51)$ & 1.16 & $(1.05-1.29)$ & 0.004 \\
\hline Model $2^{\mathrm{c}}$ & 1.00 & (Ref) & 1.19 & $(0.95-1.48)$ & 1.19 & $(0.93-1.53)$ & 1.07 & $(0.82-1.39)$ & 1.09 & $(0.98-1.22)$ & 0.108 \\
\hline \multicolumn{12}{|c|}{ Snacks and alcohol } \\
\hline Case $[n(\%)]$ & 270 & $(15.4)$ & 263 & $(15.0)$ & 234 & $(13.3)$ & 231 & (13.2) & & & \\
\hline Model $1^{\mathrm{b}}$ & 1.00 & (Ref) & 0.97 & $(0.80-1.17)$ & 0.81 & $(0.66-0.98)$ & 0.77 & $(0.63-0.95)$ & 1.00 & $(0.96-1.04)$ & 0.945 \\
\hline Model $2^{\mathrm{c}}$ & 1.00 & (Ref) & 0.94 & $(0.77-1.14)$ & 0.84 & $(0.68-1.02)$ & 0.86 & $(0.70-1.06)$ & 0.99 & $(0.96-1.03)$ & 0.655 \\
\hline \multicolumn{12}{|l|}{ White-red meat } \\
\hline Case $[n(\%)]$ & 262 & (14.9) & 242 & (13.8) & 276 & $(15.7)$ & 218 & (12.4) & & & \\
\hline Model $1^{\mathrm{b}}$ & 1.00 & (Ref) & 0.94 & $(0.78-1.14)$ & 1.05 & $(0.87-1.26)$ & 0.76 & $(0.63-0.93)$ & 0.99 & $(0.96-1.01)$ & 0.271 \\
\hline Model $2^{\mathrm{c}}$ & 1.00 & (Ref) & 0.96 & $(0.79-1.17)$ & 1.02 & $(0.84-1.23)$ & 0.81 & $(0.66-0.99)$ & 0.99 & $(0.96-1.01)$ & 0.389 \\
\hline
\end{tabular}

All values are shown numbers $(\%)$, or relative ORs (95\% CI). All estimates were derived from a multivariable logistic regression model. Bold $p$ values are statistically significant $(p<0.05)$. The range of Q1 through Q4 for each food score is shown following; $<7.1,7.1-8.9,9.0-9.8$, and $\geq 9.9$ score for grain dishes, $<2.7,2.7-4.3,4.4-6.4$, and $\geq 6.5$ score for vegetable dishes, $<4.8,4.8-7.7,7.8-9.7$, and $\geq 9.8$ score for fish and meat dishes, $<0.6,0.6-5.2,5.3-7.7$, and $\geq 7.8$ score for milk, $<1.3,1.3-3.1,3.2-5.5$, and $\geq 5.6$ score for fruits, $<8.8,8.8-9.3,9.4-9.8$, and $\geq 9.9$ score for total energy intake, $<8.7,8.7-9.5,9.6-9.8$, and $\geq 9.9$ score for snacks and alcohol, and $<5.8,5.8-8.9,9.0-9.8$, and $\geq 9.9$ score for white to red meat

$\mathrm{CI}$, confidence interval; OR, odds ratio; Ref, reference

${ }^{a}$ Linear trend $p$ values were calculated with the likelihood ratio test using the continuous variables of adherence scores

${ }^{\mathrm{b}}$ Model 1 was adjusted for age (continuous), sex (female or male), and population density ( $\geq 1000$ or $<1000$ people $/ \mathrm{km}^{2}$ )

${ }^{\mathrm{c}}$ Model 2 was Model 1 with mutual adjustment for body mass index (continuous), physical activity (yes or no), denture use (yes or no), smoking status (never smoker, past smoker, and current smoker), alcohol intake status (every day, sometimes, seldom, or never), educational attainment $(<9,10-12$, or $\geq 13$ years), medication use (continuous), living alone (yes or no), socioeconomic status (high or low), green tea consumption (frequency), coffee consumption (frequency), and history of disease (hypertension, diabetes, dyslipidaemia, heart disease, and stroke; yes or no) 
positive relationship with the prevalence of comprehensive frailty.

\section{Discussion}

Our study showed an inverse dose-response relationship between scores on adherence to the Japanese Food Guide Spinning Top and the prevalences of physical and comprehensive frailty. Higher scores of adherence to vegetable dishes and milk intake were inversely associated with both frailty types. To the best of our knowledge, this is the first study to examine the association between diet quality and prevalence of both physical and comprehensive frailty in older adults, defined by two different validation methods. Our results may corroborate the essential role of adherence to dietary guidelines in individual health.

To evaluate physical and comprehensive frailty, we used two different methods: an FP model and the KCL. The prevalence of comprehensive frailty defined by the KCL (35.8\%) was significantly higher than that defined by the FP model $(14.2 \%)$. This is consistent with previous findings [20, 36]. The people identified as physical and comprehensive frailty may not necessarily match [40]. Previous studies comparing comprehensive frailty, which considers multiple factors, and physical frailty, which focuses on physical factors only, reported that comprehensive frailty had higher accuracy in predicting the risk of mortality [41, 42]. The reason for this is that comprehensive frailty indices that assess frailty using a multi-faceted model have a positive linear correlation with age and thus reflect biological ageing [43]. Therefore, it is also needed to evaluate the association between diet quality, physical frailty, and comprehensive frailty.

In our study, higher scores on adherence were inversely associated with both physical and comprehensive frailty prevalence after adjustment for potential confounding variables. Previous studies on associations between diet quality and frailty focused mainly on the physical components [12, $13,15]$. Our findings were consistent across both types of frailty, despite several differences in indices. Despite differences in dietary measures, our findings, taken together with those from previous studies, suggest that a healthy dietary pattern is associated with a lower OR of frailty. Moreover, we also observed an inverse association between adherence scores and ORs of subdomains in the FP model and KCL. Given that the risk of the incidence of dementia and depression tends to be higher in older adults [44], it is necessary to consider the association between diet quality and physical frailty and the risk factors for comprehensive frailty. Therefore, our results may help shed more light on key dietary components in further investigations into untangling the relationship between diet and frailty.
Although it is unclear the detail of mechanism that diet quality is inversely associated with frailty prevalence, two reasons can be considered from the previous studies. The first is that some nutrient intake is negatively associated with the risk of frailty $[45,46]$. Our results indicate a positive correlation between scores on adherence and vitamin C. Actually, a previous prospective cohort study has reported that low vitamin $\mathrm{C}$ intake is associated with the risk of frailty in Spanish older adults [46]. The second is that people with high diet quality maintain better nutritional status. Many researchers have considered that healthy dietary pattern is inversely associated with the risk of the double burden of malnutrition, such as underweight and obesity [47]. We have previously reported that the lowest prevalence of both physical and comprehensive frailty was found at approximately $40 \mathrm{kcal} / \mathrm{kg}$ IBW/day (ideal body weight $=22 \times$ height $^{2}$ ) with DLW-calibrated energy intake [23] and BMI $21.4-25.7 \mathrm{~kg} /$ $\mathrm{m}^{2}$ [22]. Such facts should support our data, showing that frailty prevalence is lower among people with high diet quality. These previous studies may support our results. Although there are multiple mechanisms, such as antioxidant effects and metabolic and cardiovascular benefits, through which a healthy diet may contribute to a lower risk of frailty [14], causal relationships and detailed mechanisms must be clarified with further basic and intervention studies.

A strength of this study is that it provides additional evidence of the association between diet quality and physical and comprehensive frailty, using two validated assessment tools. These results indicate a potential robust association between diet quality and frailty. However, some methodologic limitations should be mentioned. First, our study utilised a cross-sectional study design. Therefore, no temporal or direct causal relationships of the observed associations between diet quality and frailty prevalence can be inferred. Second, although we have used previously validated FFQ $[24,25,29-31]$, given that the adherence scores to the Japanese Food Guide Spinning Top are based on specific absolute cut-offs for dietary components, it may be insufficient to evaluate the overall diet quality because we evaluated it using the FFQ, consisting of 47 food and beverage items. However, we have previously reported that the adherence scores to the Japanese Food Guide Spinning Top estimated from our used FFQ are inversely associated with the prevalence of poor oral health-related quality of life [27]. Third, owing to the observational nature of the study, we could not fully exclude residual confounding factors from self-report or unmeasured variables. For example, women and individuals with higher socioeconomic status may have greater opportunities to consume fruits, vegetables, and dairy products [48]. In the sex and socioeconomic status-stratified models, we observed an inverse association between diet quality and the prevalence of comprehensive frailty across all groups (Supplemental Tables 1 and 2). Therefore, the 
inverse association between diet quality and frailty could not be fully explained by only socioeconomic status or sex alone. Finally, our study included individuals with a history of disease, including hypertension, diabetes, and dyslipidaemia. Extrapolation to participants with attributes other than those included in the study should be performed with caution because such individuals may have received dietary advice for their disease and modified their diets accordingly. However, our results were similar after excluding participants with such diseases. Considering these limitations, rigorous longitudinal studies that further evaluate whether a healthier diet is a modifiable risk factor for frailty are needed.

In recent years, the degree of frailty increased across most adult age groups in the United States between 1999 and 2018 [49]. Our findings indicated that overall adherence scores are strongly negatively associated with ORs of frailty than any individual dietary component in the food-based Japanese dietary guidelines, and these results were similar to the previous study that reported an association between adherence to the Mediterranean diet and mortality [50]. Therefore, our results may provide useful insights for improving and preventing frailty by adherence to the food-based Japanese dietary guidelines in older adults.

In conclusion, the results of this study indicate that higher diet quality with strong adherence to the Japanese Food Guide Spinning Top is associated with a lower prevalence of both physical and comprehensive frailty. The results also show a dose-response relationship between better diet quality and a lower frailty prevalence among communitydwelling older adults. Given the rapid increase in frailty prevalence worldwide, these findings may be encouraging to older adults, who are a high-risk population for frailty.

Supplementary Information The online version contains supplementary material available at https://doi.org/10.1007/s00394-022-02819-w.

Acknowledgements We would like to thank all members of the KyotoKameoka study group for their valuable contributions. We thank Shinkan Tokudome, former Director of the National Institute of Nutrition and Health, Japan. We would like to thank Editage (www.editage.jp) for English-language editing. We thank Mayu Sugita, Yuki Okabe, Yoshizu Nozawa, Miho Ono, Tomonori Koizumi, and Hisamine Kobayashi, Ajinomoto Co., Inc., for providing funding to conduct this study.

Author contributions MK and YY: designed the research (project conception, development of overall research plan, and study oversight); TY, YW, HD, AI, KI-T, MK, and YY: conducted the research (data collection); DW and KK: analysed data or performed statistical analysis; DW, KK, HN, and CG: data interpretation; DW, KK, and YY: literature review; DW and KK: wrote the paper; DW, KK, TY, MM, and YY: had primary responsibility for final content. All authors have read and approved the final manuscript.

Funding This work was supported by a JSPS KAKENHI research grant provided to Misaka Kimura (24240091), Yosuke Yamada (15H05363), and Daiki Watanabe (21K17699). The current study was funded by
Ajinomoto Co., Inc., Tokyo, Japan. However, this study is not related to any particular products of a company, and the results do not recommend any particular products.

Availability of data and material Researchers can request our study group for permission to use these data by contacting Y.Y. (yamaday@ nibiohn.go.jp).

\section{Declarations}

Conflict of interest There are no conflicts of interests, other than those reported in funding, by any of the authors of the article.

Code availability Not applicable.

Ethics approval This study was conducted according to the guidelines laid down in the 1964 Declaration of Helsinki and its later amendments or comparable ethical standards. Ethical approval of this study was obtained from the Research Ethics Committee of Kyoto Prefectural University of Medicine (RBMR-E-363), the National Institutes of Biomedical Innovation, Health and Nutrition (NIBIOHN-76-2), and Kyoto University of Advanced Science (No. 20-1).

Consent to participate Informed consent was obtained from all individual participants included in the study.

Consent for publication Not applicable.

Open Access This article is licensed under a Creative Commons Attribution 4.0 International License, which permits use, sharing, adaptation, distribution and reproduction in any medium or format, as long as you give appropriate credit to the original author(s) and the source, provide a link to the Creative Commons licence, and indicate if changes were made. The images or other third party material in this article are included in the article's Creative Commons licence, unless indicated otherwise in a credit line to the material. If material is not included in the article's Creative Commons licence and your intended use is not permitted by statutory regulation or exceeds the permitted use, you will need to obtain permission directly from the copyright holder. To view a copy of this licence, visit http://creativecommons.org/licenses/by/4.0/.

\section{References}

1. Clegg A, Young J, Iliffe S, Rikkert MO, Rockwood K (2013) Frailty in elderly people. Lancet 381(9868):752-762. https://doi. org/10.1016/S0140-6736(12)62167-9

2. Hoogendijk EO, Afilalo J, Ensrud KE, Kowal P, Onder G, Fried LP (2019) Frailty: implications for clinical practice and public health. Lancet 394(10206):1365-1375. https://doi.org/10.1016/ S0140-6736(19)31786-6

3. Morley JE, Vellas B, van Kan GA, Anker SD, Bauer JM, Bernabei R, Cesari M, Chumlea WC, Doehner W, Evans J, Fried LP, Guralnik JM, Katz PR, Malmstrom TK, McCarter RJ, Gutierrez Robledo LM, Rockwood K, von Haehling S, Vandewoude MF, Walston J (2013) Frailty consensus: a call to action. J Am Med Dir Assoc 14(6):392-397. https://doi.org/10.1016/j.jamda.2013. 03.022

4. Lee Y, Kim J, Han ES, Ryu M, Cho Y, Chae S (2014) Frailty and body mass index as predictors of 3-year mortality in older adults living in the community. Gerontology 60(6):475-482. https://doi. org/10.1159/000362330 
5. Zaslavsky O, Zelber-Sagi S, Gray SL, LaCroix AZ, Brunner RL, Wallace RB, O'Sullivan MJ, Cochrane B, Woods NF (2016) Comparison of frailty phenotypes for prediction of mortality, incident falls, and hip fracture in older women. J Am Geriatr Soc 64(9):1858-1862. https://doi.org/10.1111/jgs.14233

6. Li G, Papaioannou A, Thabane L, Levine MAH, Ioannidis G, Wong AKO, Lau A, Adachi JD (2017) Modifying the phenotypic frailty model in predicting risk of major osteoporotic fracture in the elderly. J Am Med Dir Assoc 18(5):414-419. https://doi.org/ 10.1016/j.jamda.2016.11.015

7. Yamada M, Arai H (2015) Predictive value of frailty scores for healthy life expectancy in community-dwelling older Japanese adults. J Am Med Dir Assoc 16(11):1002e1007-1002e1011. https://doi.org/10.1016/j.jamda.2015.08.001

8. Taniguchi Y, Kitamura A, Nofuji Y, Ishizaki T, Seino S, Yokoyama Y, Shinozaki T, Murayama H, Mitsutake S, Amano H, Nishi M, Matsuyama Y, Fujiwara Y, Shinkai S (2019) Association of trajectories of higher-level functional capacity with mortality and medical and long-term care costs among community-dwelling older Japanese. J Gerontol A Biol Sci Med Sci 74(2):211-218. https://doi.org/10.1093/gerona/gly024

9. Asghari G, Mirmiran P, Yuzbashian E, Azizi F (2017) A systematic review of diet quality indices in relation to obesity. Br J Nutr 117(8):1055-1065. https://doi.org/10.1017/S0007114517000915

10. Burggraf C, Teuber R, Brosig S, Meier T (2018) Review of a priori dietary quality indices in relation to their construction criteria. Nutr Rev 76(10):747-764. https://doi.org/10.1093/nutrit/ nuy027

11. Wirt A, Collins CE (2009) Diet quality-what is it and does it matter? Public Health Nutr 12(12):2473-2492. https://doi.org/10. 1017/S136898000900531X

12. Talegawkar SA, Bandinelli S, Bandeen-Roche K, Chen P, Milaneschi Y, Tanaka T, Semba RD, Guralnik JM, Ferrucci L (2012) A higher adherence to a Mediterranean-style diet is inversely associated with the development of frailty in community-dwelling elderly men and women. J Nutr 142(12):2161-2166. https://doi. org/10.3945/jn.112.165498

13. Lopez-Garcia E, Hagan KA, Fung TT, Hu FB, Rodriguez-Artalejo F (2018) Mediterranean diet and risk of frailty syndrome among women with type 2 diabetes. Am J Clin Nutr 107(5):763-771. https://doi.org/10.1093/ajen/nqy026

14. Ward RE, Orkaby AR, Chen J, Hshieh TT, Driver JA, Gaziano JM, Djousse L (2020) Association between diet quality and frailty prevalence in the physicians' health study. J Am Geriatr Soc 68(4):770-776. https://doi.org/10.1111/jgs.16286

15. Hengeveld LM, Wijnhoven HAH, Olthof MR, Brouwer IA, Simonsick EM, Kritchevsky SB, Houston DK, Newman AB, Visser M (2019) Prospective associations of diet quality with incident frailty in older adults: the health, aging, and body composition study. J Am Geriatr Soc. https://doi.org/10.1111/jgs.16011

16. Yoshiike N, Hayashi F, Takemi Y, Mizoguchi K, Seino F (2007) A new food guide in Japan: the Japanese Food Guide Spinning Top. Nutr Rev 65(4):149-154. https://doi.org/10.1111/j.1753-4887. 2007.tb00294.x

17. Kurotani K, Akter S, Kashino I, Goto A, Mizoue T, Noda M, Sasazuki S, Sawada N, Tsugane S, Japan Public Health Center based Prospective Study G (2016) Quality of diet and mortality among Japanese men and women: Japan Public Health Center based prospective study. BMJ 352:i1209. https://doi.org/10.1136/ bmj.i1209

18. Dent E, Lien C, Lim WS, Wong WC, Wong CH, Ng TP, Woo J, Dong B, de la Vega S, Hua Poi PJ, Kamaruzzaman SBB, Won C, Chen LK, Rockwood K, Arai H, Rodriguez-Manas L, Cao L, Cesari M, Chan P, Leung E, Landi F, Fried LP, Morley JE, Vellas B, Flicker L (2017) The Asia-Pacific clinical practice guidelines for the management of frailty. J Am Med Dir Assoc 18(7):564575. https://doi.org/10.1016/j.jamda.2017.04.018

19. Hewitt J, Carter B, Vilches-Moraga A, Quinn TJ, Braude P, Verduri A, Pearce L, Stechman M, Short R, Price A, Collins JT, Bruce E, Einarsson A, Rickard F, Mitchell E, Holloway M, Hesford J, Barlow-Pay F, Clini E, Myint PK, Moug SJ, McCarthy K, Collaborators CS (2020) The effect of frailty on survival in patients with COVID-19 (COPE): a multicentre, European, observational cohort study. Lancet Public Health 5(8):e444-e451. https://doi. org/10.1016/S2468-2667(20)30146-8

20. Yamada Y, Nanri H, Watanabe Y, Yoshida T, Yokoyama K, Itoi A, Date H, Yamaguchi M, Miyake M, Yamagata E, Tamiya H, Nishimura M, Fujibayashi M, Ebine N, Yoshida M, Kikutani T, Yoshimura E, Ishikawa-Takata K, Yamada M, Nakaya T, Yoshinaka Y, Fujiwara Y, Arai H, Kimura M (2017) Prevalence of frailty assessed by Fried and Kihon checklist indexes in a prospective cohort study: design and demographics of the Kyoto-kameoka longitudinal study. J Am Med Dir Assoc 18(8):733e737-733e715. https://doi.org/10.1016/j.jamda.2017. 02.022

21. Watanabe D, Yoshida T, Watanabe Y, Yamada Y, Kimura M, Group KS (2020) Objectively measured daily step counts and prevalence of frailty in 3,616 older adults. J Am Geriatr Soc 68(10):2310-2318. https://doi.org/10.1111/jgs.16655

22. Watanabe D, Yoshida T, Watanabe Y, Yamada Y, Kimura M, Kyoto-Kameoka Study G (2020) A U-shaped relationship between the prevalence of frailty and body mass index in communitydwelling Japanese older adults: The Kyoto-Kameoka study. J Clin Med. https://doi.org/10.3390/jcm9051367

23. Watanabe D, Yoshida T, Nanri H, Watanabe Y, Date H, Itoi A, Goto C, Ishikawa-Takata K, Sagayama H, Ebine N, Kobayashi H, Kimura M, Yamada Y, for Kyoto-Kameoka S, (2021) Association between the prevalence of frailty and doubly labeled watercalibrated energy intake among community-dwelling older adults. J Gerontol A Biol Sci Med Sci 76(5):876-884. https://doi.org/10. 1093/gerona/glaa133

24. Watanabe D, Nanri H, Yoshida T, Yamaguchi M, Sugita M, Nozawa Y, Okabe Y, Itoi A, Goto C, Yamada Y, Ishikawa-Takata K, Kobayashi H, Kimura M, Kyoto-Kameoka Study Group KS (2019) Validation of energy and nutrition intake in Japanese elderly individuals estimated based on a short food frequency questionnaire compared against a 7-day dietary record: the KyotoKameoka study. Nutrients. https://doi.org/10.3390/nu11030688

25. Watanabe D, Nanri H, Sagayama H, Yoshida T, Itoi A, Yamaguchi M, Yokoyama K, Watanabe Y, Goto C, Ebine N, Higaki Y, Ishikawa-Takata K, Kimura M, Yamada Y, Kyoto-Kameoka Study G (2019) Estimation of energy intake by a food frequency questionnaire: calibration and validation with the doubly labeled water method in Japanese older people. Nutrients. https://doi.org/ 10.3390/nu11071546

26. Watanabe D, Yoshida T, Yokoyama K, Yoshinaka Y, Watanabe Y, Kikutani T, Yoshida M, Yamada Y, Kimura M, Kyoto-Kameoka Study G (2020) Association between mixing ability of masticatory functions measured using color-changing chewing gum and frailty among Japanese older adults: the Kyoto-Kameoka study. Int J Environ Res Public Health. https://doi.org/10.3390/ijerph1712 4555

27. Watanabe D, Kurotani K, Yoshida T, Nanri H, Watanabe Y, Date H, Itoi A, Goto C, Ishikawa-Takata K, Kikutani T, Yoshida M, Fujita H, Yamada Y, Kimura M, Kyoto-Kameoka Study G (2021) Adherence to the food-based Japanese dietary guidelines and prevalence of poor oral health-related quality of life among older Japanese adults in the Kyoto-Kameoka study. Br J Nutr. https:// doi.org/10.1017/S0007114521003329

28. Lachat C, Hawwash D, Ocke MC, Berg C, Forsum E, Hornell A, Larsson C, Sonestedt E, Wirfalt E, Akesson A, Kolsteren P, 
Byrnes G, De Keyzer W, Van Camp J, Cade JE, Slimani N, Cevallos M, Egger M, Huybrechts I (2016) Strengthening the reporting of observational studies in epidemiology-nutritional epidemiology (STROBE-nut): an extension of the STROBE statement. PLoS Med 13(6):e1002036. https://doi.org/10.1371/journal.pmed.10020 36

29. Imaeda N, Goto C, Sasakabe T, Mikami H, Oze I, Hosono A, Naito M, Miyagawa N, Ozaki E, Ikezaki H, Nanri H, Nakahata NT, Kamano SK, Kuriki K, Yaguchi YT, Kayama T, Kurihara A, Harada S, Wakai K (2021) Reproducibility and validity of food group intake in a short food frequency questionnaire for the middle-aged Japanese population. Environ Health Prev Med 26(1):28. https://doi.org/10.1186/s12199-021-00951-3

30. Tokudome S, Goto C, Imaeda N, Tokudome Y, Ikeda M, Maki S (2004) Development of a data-based short food frequency questionnaire for assessing nutrient intake by middle-aged Japanese. Asian Pac J Cancer Prev 5(1):40-43

31. Tokudome Y, Goto C, Imaeda N, Hasegawa T, Kato R, Hirose K, Tajima K, Tokudome S (2005) Relative validity of a short food frequency questionnaire for assessing nutrient intake versus threeday weighed diet records in middle-aged Japanese. J Epidemiol 15(4):135-145. https://doi.org/10.2188/jea.15.135

32. Watanabe D, Yoshida T, Yoshimura E, Nanri H, Goto C, Ishikawa-Takata K, Ebine N, Fujita H, Kimura M, Yamada Y, KyotoKameoka Study G (2021) Doubly labelled water-calibration approach attenuates the underestimation of energy intake calculated from self-reported dietary assessment data in Japanese older adults. Public Health Nutr. https://doi.org/10.1017/S136898002 1003785

33. Council for Science and Technology; Ministry of Education, Culture, Sports, Science and Technology, Japan (2001) Standard tables of food composition in Japan, fifth revised and enlarged edition. Tokyo, National Printing Bureau (In Japanese)

34. Oba S, Nagata C, Nakamura K, Fujii K, Kawachi T, Takatsuka N, Shimizu H (2009) Diet based on the Japanese Food Guide Spinning Top and subsequent mortality among men and women in a general Japanese population. J Am Diet Assoc 109(9):1540-1547. https://doi.org/10.1016/j.jada.2009.06.367

35. Satake S, Shimokata H, Senda K, Kondo I, Toba K (2017) Validity of total Kihon checklist score for predicting the incidence of 3 -year dependency and mortality in a community-dwelling older population. J Am Med Dir Assoc 18(6):552e551-552e556. https:// doi.org/10.1016/j.jamda.2017.03.013

36. Ambagtsheer RC, Visvanathan R, Dent E, Yu S, Schultz TJ, Beilby J (2020) Commonly used screening instruments to identify frailty among community-dwelling older people in a general practice (primary care) setting: a study of diagnostic test accuracy. J Gerontol A Biol Sci Med Sci 75(6):1134-1142. https://doi.org/ 10.1093/gerona/glz260

37. van Buuren S, Groothuis-Oudshoorn K (2011) Multivariate imputation by chained equations in R. J Stat Softw 45:1-67

38. Willett W, Stampfer MJ (1986) Total energy intake: implications for epidemiologic analyses. Am J Epidemiol 124(1):17-27. https://doi.org/10.1093/oxfordjournals.aje.a114366
39. Darmon N, Drewnowski A (2008) Does social class predict diet quality? Am J Clin Nutr 87(5):1107-1117. https://doi.org/10. 1093/ajen/87.5.1107

40. Lin SM, Aliberti MJR, Fortes-Filho SQ, Melo JA, Aprahamian I, Suemoto CK, Jacob Filho W (2018) Comparison of 3 frailty instruments in a geriatric acute care setting in a low-middle income country. J Am Med Dir Assoc 19(4):310e313-314e313. https://doi.org/10.1016/j.jamda.2017.10.017

41. Rockwood K, Andrew M, Mitnitski A (2007) A comparison of two approaches to measuring frailty in elderly people. J Gerontol A Biol Sci Med Sci 62(7):738-743. https://doi.org/10.1093/ gerona/62.7.738

42. Kulminski AM, Ukraintseva SV, Kulminskaya IV, Arbeev KG, Land K, Yashin AI (2008) Cumulative deficits better characterize susceptibility to death in elderly people than phenotypic frailty: lessons from the Cardiovascular Health Study. J Am Geriatr Soc 56(5):898-903. https://doi.org/10.1111/j.1532-5415.2008.01656.x

43. Rockwood K, Rockwood MR, Mitnitski A (2010) Physiological redundancy in older adults in relation to the change with age in the slope of a frailty index. J Am Geriatr Soc 58(2):318-323. https:// doi.org/10.1111/j.1532-5415.2009.02667.x

44. Byers AL, Yaffe K (2011) Depression and risk of developing dementia. Nat Rev Neurol 7(6):323-331. https://doi.org/10.1038/ nrneurol.2011.60

45. Bartali B, Frongillo EA, Bandinelli S, Lauretani F, Semba RD, Fried LP, Ferrucci L (2006) Low nutrient intake is an essential component of frailty in older persons. J Gerontol A Biol Sci Med Sci 61(6):589-593. https://doi.org/10.1093/gerona/61.6.589

46. Balboa-Castillo T, Struijk EA, Lopez-Garcia E, Banegas JR, Rodriguez-Artalejo F, Guallar-Castillon P (2018) Low vitamin intake is associated with risk of frailty in older adults. Age Ageing 47(6):872-879. https://doi.org/10.1093/ageing/afy 105

47. Collaborators GBDD (2019) Health effects of dietary risks in 195 countries, 1990-2017: a systematic analysis for the Global Burden of Disease Study 2017. Lancet 393(10184):1958-1972. https:// doi.org/10.1016/S0140-6736(19)30041-8

48. Okubo H, Murakami K, Sasaki S (2016) Monetary value of self-reported diets and associations with sociodemographic characteristics and dietary intake among Japanese adults: analysis of nationally representative surveys. Public Health Nutr 19(18):3306-3318. https://doi.org/10.1017/S1368980016001695

49. Joanna M, Blodgett KR, Theou O (2021) Changes in the severity and lethality of age-related health deficit accumulation in the USA between 1999 and 2018: a population-based cohort study. Lancet Healthy Longev 2(2):e96-104

50. Buckland G, Agudo A, Travier N, Huerta JM, Cirera L, Tormo MJ, Navarro C, Chirlaque MD, Moreno-Iribas C, Ardanaz E, Barricarte A, Etxeberria J, Marin P, Quiros JR, Redondo ML, Larranaga N, Amiano P, Dorronsoro M, Arriola L, Basterretxea M, Sanchez MJ, Molina E, Gonzalez CA (2011) Adherence to the Mediterranean diet reduces mortality in the Spanish cohort of the European Prospective Investigation into Cancer and Nutrition (EPIC-Spain). Br J Nutr 106(10):1581-1591. https://doi.org/10. 1017/S0007114511002078 


\section{Authors and Affiliations}

\section{Daiki Watanabe ${ }^{1,2} \cdot$ Kayo Kurotani $^{1,3} \cdot$ Tsukasa Yoshida $^{1,2} \cdot$ Hinako Nanri $^{1} \cdot$ Yuya Watanabe ${ }^{1,2,4} \cdot$ Heiwa Date $^{5}$. Aya Itoi ${ }^{1,6} \cdot$ Chiho Goto $^{7}$ Kazuko Ishikawa-Takata ${ }^{1,8} \cdot$ Misaka Kimura ${ }^{2,9,10} \cdot$ Motohiko Miyachi $^{1,11}$. Yosuke Yamada $^{1,2}$ (D) Kyoto-Kameoka Study Group ${ }^{1}$}

Daiki Watanabe

d2watanabe@nibiohn.go.jp

Kayo Kurotani

k-kurotani@swu.ac.jp

Tsukasa Yoshida

t-yoshida@nibiohn.go.jp

Hinako Nanri

hnanri@nibiohn.go.jp

Yuya Watanabe

yu15-watanabe@my-zaidan.or.jp

Heiwa Date

heiwa.date@biwako.shiga-u.ac.jp

Aya Itoi

aitoi@kwjc.kobe-wu.ac.jp

Chiho Goto

goto.chiho@nagoya-bunri.ac.jp

Kazuko Ishikawa-Takata

kt207460@nodai.ac.jp

Misaka Kimura

kimura.misaka@kuas.ac.jp

Motohiko Miyachi

cardiovascular0327@mac.com

1 Department of Physical Activity Research, National Institute of Health and Nutrition, National Institutes of Biomedical Innovation, Health and Nutrition, 1-23-1 Toyama,

Shinjuku-ku, Tokyo 162-8636, Japan
2 Institute for Active Health, Institute of Interdisciplinary Research, Kyoto University of Advanced Science, Kyoto 621-8555, Japan

3 Faculty of Food and Health Sciences, Showa Women's University, Tokyo 154-8533, Japan

4 Physical Fitness Research Institute, Meiji Yasuda Life Foundation of Health and Welfare, Tokyo 192-0001, Japan

5 Department of Data Science, Shiga University, Shiga 522-8522, Japan

6 Department of Health, Sports and Nutrition, Faculty of Health and Welfare, Kobe Women's University, Hyogo 650-0046, Japan

7 Department of Health and Nutrition, Faculty of Health and Human Life, Nagoya Bunri University, Aichi 492-8520, Japan

8 Faculty of Applied Biosciences, Tokyo University of Agriculture, Tokyo 156-8502, Japan

9 Laboratory of Applied Health Sciences, Kyoto Prefectural University of Medicine, Kyoto 602-8566, Japan

10 Department of Nursing, Doshisha Women's College of Liberal Arts, Kyoto 610-0394, Japan

11 Faculty of Sport Sciences, Waseda University, Saitama 359-1192, Japan 